\title{
VALIDAÇÃO DE MÉTODO ANALÍTICO PARA QUANTIFICAÇÃO DO FIPRONIL POR CROMATOGRAFIA LÍQUIDA DE ALTA EFICIÊNCIA
}

Fausto de Souza Pagan¹, Alexandre de Faria Lima ${ }^{2}$, Urias Fernandes Maciel Junior ${ }^{3}$, Deusmaque Carneiro Ferreira ${ }^{4}$

${ }^{1}$ Doutorando em Química pelo Programa de Pós-Graduação Multicêntrico em Química de Minas Gerais da Universidade Federal do Triângulo Mineiro, Uberaba, Minas Gerais, Brasil.

${ }^{2}$ Professor Doutor do Curso de Engenharia Química da Universidade de Uberaba, Minas Gerais, Brasil.

${ }^{3}$ Engenheiro Químico pela Universidade de Uberaba, Minas Gerais, Brasil.

${ }^{4}$ Professor Doutor do Programa de Pós-Graduação em Ciência e Tecnologia Ambiental da Universidade Federal do Triângulo Mineiro, Uberaba, Minas Gerais, Brasil (E-mail:deusmaque.ferreira@uftm.edu.br).

\section{Recebido em: 06/04/2019 - Aprovado em: 10/06/2019 - Publicado em: 30/06/2019} DOI: 10.18677/EnciBio_2019A188

\begin{abstract}
RESUMO
A demanda por métodos analíticos precisos e confiáveis é fator comum nas últimas décadas de avanço das tecnologias e dos modos de produção. Inúmeros estudos para testes analíticos foram propostos criando diversos parâmetros de respostas que levavam a conflitos de interpretação quando comparados. Para permitir a possibilidade de comparação e verificação de confiabilidade das respostas obtidas nestes testes, órgãos e instituições determinam orientações e regulam diretrizes que permitem validar os métodos certificando-os como parâmetros metodológicos a serem seguidos. No Brasil, ANVISA e INMETRO regulamentam os testes de validação de metodologias analíticas. A cromatografia líquida de alta eficiência (CLAE) é fonte de pesquisa em metodologias analíticas para identificação de compostos, dentre estes aqueles voltados para o ramo dos fármacos e pesticidas. $O$ objetivo do presente estudo foi validar uma metodologia analítica para quantificação do princípio ativo do Fipronil por meio de CLAE com detector ultravioleta. Em relação aos parâmetros analíticos empregados para a validação, a linearidade verificada para o método apresentou valor de $r=0,9999$ sendo obtida a equação do ajuste por meio da regressão linear em mínimos quadrados. Os limites de detecção e quantificação determinados foram $0,128 \mathrm{mg} \cdot \mathrm{L}^{-1}$ e $0,426 \mathrm{mg} \cdot \mathrm{L}^{-1}$, respectivamente. $A$ repetitividade e reprodutibilidade, também verificadas para o método analítico, retornaram desvio-padrão residual dentro dos limites estabelecidos pela norma e consonantes para os verificados na literatura corroborando para validação e robustez do método avaliado.
\end{abstract}

PALAVRAS-CHAVE: melhoria contínua, produtividade, validação analítica. 


\title{
VALIDATION OF ANALYTICAL METHOD FOR QUANTIFICATION OF FIPRONYL BY HIGH PERFOMANCE LIQUID CHROMATOGRAPHY
}

\begin{abstract}
The demand for accurate and reliable analytical methods is a common factor in the last decades of technology advancement and modes of production. Numerous studies for analytical tests were proposed, creating several parameters of answers that led to conflicts of interpretation when compared. To allow for the possibility of comparison and reliability verification of the answers obtained in these tests, agencies and institutions determine guidelines and regulate guidelines that allow validating the methods, certifying them as methodological parameters to be followed. In Brazil, ANVISA and INMETRO regulate the validation tests of analytical methodologies. High performance liquid chromatography (HPLC) is a source of research in analytical methodologies for the identification of compounds, among them those directed to the branch of drugs and pesticides. The aim of the present study was to validate an analytical methodology for quantification of the active principle of Fipronil by HPLC with ultraviolet detector. In relation to the analytical parameters used for the validation, the linearity verified for the method presented a value of $r=0.9999$ and the equation of the adjustment was obtained by linear regression in least squares. The limits of detection and quantification were 0.128 $\mathrm{mg} \cdot \mathrm{L}^{-1}$ and $0.426 \mathrm{mg} \cdot \mathrm{L}^{-1}$, respectively. The repeatability and reproducibility, also verified for the analytical method, returned residual standard deviation within the limits established by the standard and consonants for those verified in the literature corroborating for validation and robustness of the evaluated method.
\end{abstract}

KEYWORDS: continuous improvement, productivity, analytical validation.

\section{INTRODUÇÃO}

A busca por metodologias analíticas que sejam confiáveis, comparáveis e reproduzíveis em ampla escala tem sido alvo de pesquisas constantes nos ramos da química qualitativa e quantitativa (CHELLINI et al., 2015). Para que uma metodologia analítica de identificação ou mesmo quantificação seja difundida como parâmetro de determinação para qualidade e certeza de resultados ela deve passar por um sistema de testes e verificações denominado validação (SWARTZ ; IRA, 2018).

Para orientar a validação os órgãos ou agências responsáveis elaboram diretrizes que devem ser seguidas pelos laboratórios e analistas quando na execução de metodologias próprias de uso analítico (COELHO et al., 2018). Tais diretrizes, previamente estudadas e exaustivamente verificadas, quando adotadas pelos laboratórios fornecem aos órgãos a garantia de que os dados por eles produzidos são corroborados pelas próprias técnicas propostas e que carregam a confiabilidade da certificação estabelecida (NOVAES et al., 2017).

Os processos de validação se subdividem em duas classes principais, uma determinada de validação no laboratório, que consistem em técnicas de apuração da metodologia levando-se em consideração os inúmeros dados coletados no mesmo laboratório, com mesmo equipamento, analistas e condições repetidas do meio (CANDIOTI et al., 2014). A segunda classe, chamada validação completa, consiste na verificação do método em diferentes laboratórios que integram uma rede análoga de determinação na qual o método de verificação será o mesmo, mas as condições laboratoriais, analistas e equipamentos serão diferentes (BENEVIDES et al., 2014). 
A consolidação do método se dá quando a validação no laboratório e a completa reproduzem resultados consonantes (RIBANI et al., 2004).

No Brasil, os órgãos reguladores responsáveis pela validação de metodologias analíticas são a Agência Nacional de Vigilância Sanitária (ANVISA), mais voltada para o ramo alimentício e farmacêutico e o Instituto Nacional de Metrologia, Qualidade e Tecnologia (INMETRO, 2018). Para a ANVISA a diretriz de validação de métodos analíticos (DAE) dada pela Resolução da Diretoria Colegiada, RDC $n^{\circ} 166$ de 24 de julho de 2017 (ANVISA, 2017). Para o INMETRO a orientação fica a cargo do documento DOQ-CGCRE-008 de 05 de agosto de 2016 atualizado em julho de 2018 (INMETRO, 2018).

Pelas disposições vigentes, o processo de validação passa por uma série de ensaios que incluem, dentre outros, linearidade, limite de detecção (LD), limite de quantificação (LQ), seletividade, repetitividade, reprodutibilidade, robustez e outros parâmetros estatísticos de análise descritiva (INMETRO, 2018; ANVISA, 2017).

A linearidade busca identificar um comportamento diretamente proporcional entre a variável medida e uma resposta dentro de um intervalo determinado (BARROS et al., 2002). Geralmente desconhecido antes do teste, o comportamento pode ser verificado empiricamente através da obtenção de uma curva analítica em que os dados obtidos são levados à regressão linear resultando em um equacionamento descritivo do comportamento observado (DURAND et al., 2016).

A regressão linear é bem posta quando o seu coeficiente de correlação, $r$, se aproxima do valor unitário. A ANVISA recomenda valores de $r$ superiores a 0,99 enquanto o INMETRO, superiores a 0,90 (ANVISA, 2017; INMETRO, 2018). Outros parâmetros constituem ponto de relevante interesse para análise de linearidade como o desvio-padrão e o desvio-padrão residual (DPR) dos dados coletados (NOVAES et al., 2017).

O parâmetro LD indica o menor valor de uma variável (concentração) que pode ser detectado, porém não necessariamente quantificado para os limites de exatidão e precisão exigidos pelo método (SANTOS et al., 2014). Já o parâmetro LQ indica a menor quantificação possível para uma variável dentro de um determinado método avaliado (CASSINI et al., 2013). Em trabalhos de validação de metodologia analítica o LD e LQ são comumente obtidos com base nos parâmetros da curva analítica ou na razão sinal e ruído gerados nos equipamentos eletrônicos (SANTOS, 2018).

A seletividade é o parâmetro responsável pala certeza da identificação pelo método para a variável de resposta trabalhada, obtendo assim a relação de impurezas e interferentes que possam gerar identificações falsas no método estudado (IAMMARINO et al., 2017). Esse parâmetro deve ser continuamente verificado no decorrer dos testes para os demais parâmetros (GOES JUNIOR et al., 2019).

A repetitividade busca verificar a concordância dos valores coletados para uma metodologia obtidos nas mesmas condições de laboratório, equipamento, operadores entre outras variáveis que possam influenciar o método enquanto que a reprodutibilidade busca a concordância dos resultados obtidos para a mesma amostra e método, mas em condições distintas como equipamentos de mesmo modelo, porém diferentes analistas e laboratórios distintos (CHAWLA et al., 2016; SANTOS, 2018). Ambos os parâmetros devem encontrar seus limites de divergência 
dentro dos resultados aceitáveis segundo metodologia de validação seguida (AGUIAR JÚNIOR et al., 2018).

Pequenas variações são impossíveis de serem excluídas dos testes estando presentes tanto na repetitividade quanto na reprodutibilidade, gerando os erros aleatórios (AGUIAR JÚNIOR et al., 2018). Como forma de relativizar essas divergências o parâmetro robustez mede a sensibilidade do método analítico frente a essas variações (LEGRAND et al., 2018).

Uma boa robustez indica que o método mantém-se confiável e exato frente às oscilações. Nos testes de validação a robustez é avaliada por meio de uma pequena variação de parâmetros conhecidos, sendo então verificada a sua influência na resposta (IAMMARINO et al., 2017). Vale destacar que uma metodologia descritiva pode ser obtida pelo teste de Youden recomendada pelo INMETRO (INMETRO, 2018).

Uma fonte de grande obtenção de metodologias analíticas é a Cromatografia Líquida de Alta Eficiência (CLAE), do inglês "High Performance Liquid Chromatography" (HPLC) (GOUTAL et al., 2016).

A cromatografia trabalha na separação dos compostos presentes em uma matriz de acordo com seu tempo de retenção ou eluição, influenciado pela interação da amostra, arrastada pela fase móvel, com a fase estacionária que preenche uma coluna capilar, caso o fluido de arraste seja um líquido tem-se a cromatografia líquida, caso seja um gás, cromatografia gasosa (QI, et al., 2015; LUCIANIGIACOBBE et al., 2018).

O método cromatográfico depende também dos detectores, sendo os mais comuns o ultravioleta (UV), arranjo de fotodiodos e o espectrômetro de massas que quando associados à cromatografia formam um sistema analítico denominado hifenado (LEGRAND et al., 2018).

Os detectores UV são amplamente utilizados na cromatografia líquida apresentando boas faixas de detecção (sensibilidade de aproximadamente $10^{-9}$ g. $\mathrm{mL}^{-1}$ ) e robustez enquanto são viáveis economicamente (SCHWEIKART \& HULTHE, 2019). Esses detectores relacionam a absorção do composto com a sua concentração, lei de Lambert- Beer (LEGRAND et al., 2018).

A HPLC/UV é amplamente utilizada na detecção e determinação de inúmeros composto e ensaios analíticos encontrando forte aplicação para a indústria farmacêutica e de pesticidas que encontram no método a descrição completa das formulações e suas propriedades químicas (OHIRA et al., 2018). Como exemplo de composto analisado por HPLC tem-se o Fipronil.

O Fipronil foi desenvolvido pela Rhone-Poulenc e comercializado atualmente pela BASF®, trata-se de um pesticida da família dos pirazois que apresenta como princípio ativo o fenilpirazol, classe II, muito tóxico, que age por contato ou ingestão. Nos insetos ele inibe a captação de cloretos nos receptores gabaérgicos e canais glutamato-cloro, estes são específicos da classe Insecta evidenciando a seletividade do referido pesticida (GUPTA \& ANADÓN, 2018). Os íons cloretos não captados nos receptores irão acumular, essa diferença de concentração dentro e fora da célula provoca uma super excitação no Sistema Nervoso Central (SNC) resultando na neurastenia e a consequente morte do inseto (HOLDER et al., 2018). A Figura 1 ilustra a estrutura química do fenilpirazol. 


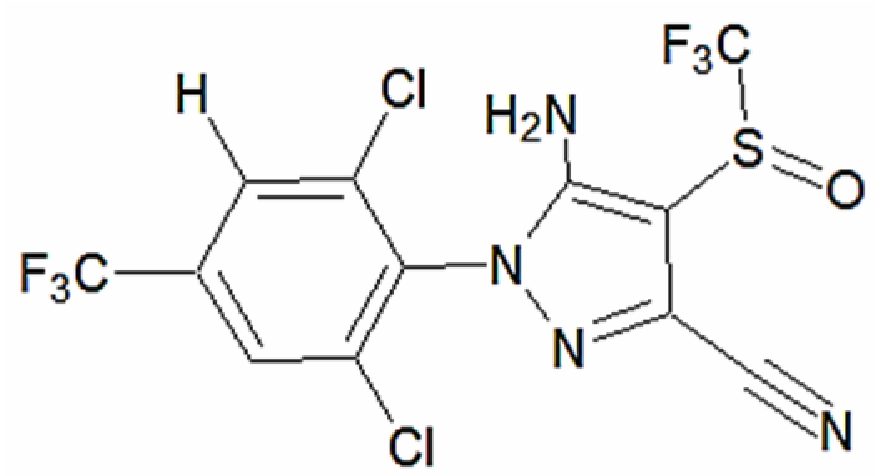

FIGURA 1- Molécula do fenilpirazol, nomenclatura IUPAC: (RS)-5-amino-1[2,6-dichloro-4-(trifluormethyl)phenyl]-4-(trifluormethylsulfinyl)-1H-pyrazole-3carbonitrile.

Fonte: Os autores, (2019)

Ante a importância da validação de metodologia analítica para confiabilidade dos dados somada a constante utilização dos pesticidas do grupo pirazol, o objetivo do presente estudo foi validar a metodologia analítica para quantificação do princípio ativo do Fipronil por meio da cromatografia líquida de alta eficiência com detector UV.

\section{MATERIAL E MÉTODOS}

Todas as soluções foram preparadas imediatamente antes de cada procedimento experimental e todos os reagentes químicos utilizados neste trabalho foram P.A, utilizados conforme recebidos.

A análise da concentração do princípio ativo (p.a.) no Fipronil 96,11\% (m/m) foi realizada solubilizando-se $62,5 \mathrm{mg}$ do produto em metanol via banho ultrassônico durante dez minutos. Após solubilização o volume foi aferido a $100 \mathrm{~mL}$ em balão volumétrico. A solução então foi analisada via HPLC de marca Shimadzu, detector

UV operando em comprimento de onda de $254 \mathrm{~nm}$, coluna C18 (250 mm x 4,6 mm x

$5 \mu \mathrm{m})$, temperatura de operação do forno $25^{\circ} \mathrm{C}$, utilizando como fase móvel mistura

1:3 água:acetonitrila em fluxo de $1 \mathrm{~mL} \cdot \mathrm{min}^{-1}$ e volume de injeção da amostra de $5 \mu \mathrm{L}$.

Para validação da metodologia analítica de determinação do princípio ativo do Fipronil foram determinados os parâmetros comuns adotados pelos órgãos reguladores no âmbito nacional, INMETRO e ANVISA, como a linearidade; LD; LQ; seletividade; repetitividade; reprodutibilidade; robustez e parâmetros estatísticos 
descritivos (média, desvio-padrão, DPR, recuperação, sensibilidade, estabilidade, coeficientes de correlação e determinação e teste de Grubbs).

A linearidade foi estabelecida mediante a curva analítica obtida através da medição, em triplicata, da concentração do Fipronil 96,11\% em diferentes soluções levadas a teste em HPLC/UV e seguinte dispersão dos valores em um gráfico. Para a curva, sete soluções foram preparadas pesando-se 14,$7 ; 20,4 ; 26,3 ; 31,7 ; 35,8$; 41,1 e $47,7 \mathrm{mg}$ do Fipronil $96,11 \%$ e solubilizadas em metanol, via banho ultrassônico por dez minutos, ajustando posteriormente o volume a $100 \mathrm{~mL}$ aferidos em balão volumétrico.

Os parâmetros LD e LQ foram determinados via metodologia da razão sinal e ruído do método HPLC/UV juntamente com os dados da curva analítica obtida na linearidade. A solução de menor concentração preparada para a curva foi inserida no cromatógrafo e posteriormente o branco da amostra originando as medições em que para o LD a razão esperada deverá ser menor que três, enquanto que para o LQ a razão deverá ser menor que dez conforme orienta o INMETRO.

As Equações 1 e 2 descrevem, respectivamente, a determinação do LD e LQ segundo resolução vigente (ANIVSA, 2017).

$$
\begin{array}{ll}
L D=\frac{3,3 \cdot \mathrm{DPR}}{\mathrm{IC}} & \text { Equação 1 } \\
L Q=\frac{10 \cdot \mathrm{DPR}}{\mathrm{IC}} & \text { Equação 2 }
\end{array}
$$

Em que:

LD = limite de detecção do método para o analito;

$\mathrm{LQ}=$ limite de quantificação do método para o analito;

$\mathrm{DPR}=$ desvio padrão residual da curva analítica obtida;

$I C=$ coeficiente angular da curva analítica obtida, considerando a regressão linear ajustada;

A seletividade e recuperação, parâmetros que corroboram com a exatidão da metodologia de identificação do Fipronil $96,11 \%$, foram avaliadas por meio da inserção de amostras, placebo e branco analítico no HPLC/UV avaliando as condições de eluição da amostra, os tempos de retenção e a possível existência de interferentes verificados no cromatograma de absorção do Fipronil.

Guardadas as mesmas condições dos ensaios anteriores, ambiente, operador, operação e sistemática de testes, a repetitividade foi determinada através de medição do p.a. do Fipronil para sete diferentes soluções preparadas pesando-se 58,$9 ; 60,1 ; 61,8 ; 65,3 ; 66,4$ e $69,6 \mathrm{mg}$ da amostra e solubilizadas em $100 \mathrm{~mL}$ de metanol via banho ultrassônico por dez minutos.

Com o HPLC/UV em condições de equilíbrio de uso as amostras foram inseridas, em triplicata, sendo identificadas as concentrações de Fipronil totalizando 21 medições para a repetição metodológica.

Correlata a repetitividade, a reprodutibilidade referente a variações internas do mesmo laboratório, foi determinada via medição da concentração de Fipronil 96,11\% por HPLC/UV em 14 soluções preparadas e medidas em duplicata por dois analistas distintos, cada um responsável por sete amostras em medição. 
O preparo das soluções seguiu metodologia de preparo análoga aquela anteriormente descrita para o teste de repetitividade. Para o analista 01 as massas de Fipronil 96,11\% usada nos preparos foi de 58,9;60,1;61,1;61,8;65,3;66,4 e 69,6 mg e para o analista 02 massas de 55,6; 57,4; 59,8; 63,4; 64,4; 65,2 e 71,7 mg solubilizadas em metanol e perfazendo $100 \mathrm{~mL}$ de cada solução analisadas por cada analista.

Para verificar a robustez da metodologia analítica investigada escolheu-se para a variável de interferência a temperatura do forno. As sete amostras preparadas para o ensaio de repetitividade foram novamente analisadas em duas novas condições de temperatura, $20^{\circ} \mathrm{C}$ e $30^{\circ} \mathrm{C}$, além daquela adotada anteriormente nos demais testes como sendo $25^{\circ} \mathrm{C}$.

Os parâmetros lineares de trabalho para verificação da estabilidade dos dados anteriores foram determinados via metodologia estatística para determinação de média, desvio-padrão, DPR, recuperação, estabilidade, coeficientes angular, linear, de determinação e de correlação, teste estatístico de Grubbs e limite de confiança conforme apresentados nos resultados.

\section{RESULTADOS E DISCUSSÃO}

O parâmetro da linearidade verificado foi avaliado de acordo com a área dos picos, obtida pelos cromatogramas referentes ao princípio ativo do Fipronil em função da concentração da solução inicial. Os valores de trabalho estão apresentados na Tabela 1. Obteve-se para o coeficiente de determinação, $\mathrm{R}^{2}$, o valor de 0,9998 , ou seja, $99,98 \%$ dos dados de concentração de Fipronil são preditos pelo equacionamento obtido da regressão linear apresentado na Equação 3. A resposta do detector foi linear à concentração do Fipronil na faixa de 0,141 a $0,458 \mathrm{mg} \cdot \mathrm{mL}^{-1}$.

$$
f(x)=2,9475.10^{6} x+4071,1 \quad \text { Equação } 3
$$

, onde:

$\mathrm{f}(\mathrm{x})$ = área dos picos referentes ao princípio ativo do Fipronil; $\mathrm{x}=$ concentração da solução de Fipronil $99,11 \%, \mathrm{mg} \cdot \mathrm{mL}^{-1}$.

TABELA 1- Valores de concentração e área do pico referentes ao Fipronil obtidos do cromatograma.

\begin{tabular}{ccc}
\hline Amostra & $\begin{array}{c}\text { Concentração do Fipronil } \\
\left(\mathbf{m g}_{\mathbf{m}}^{-1}\right)\end{array}$ & $\begin{array}{c}\text { Área do pico } \\
\text { (ua) }\end{array}$ \\
\hline 7 & 0,458 & 1360023,00 \\
6 & 0,395 & 1167043,00 \\
5 & 0,344 & 1016954,50 \\
4 & 0,305 & 892207,33 \\
3 & 0,253 & 751446,33 \\
2 & 0,196 & 586391,33 \\
1 & 0,141 & 420697,00 \\
\hline
\end{tabular}

A Figura 2 apresenta o gráfico de linha dos pontos da área dos picos referentes ao princípio ativo do Fipronil em função da concentração da solução inicial juntamente com os dados da regressão linear conforme Resolução $n^{\circ} 166$ de 24 de julho de 2017 da ANVISA. 


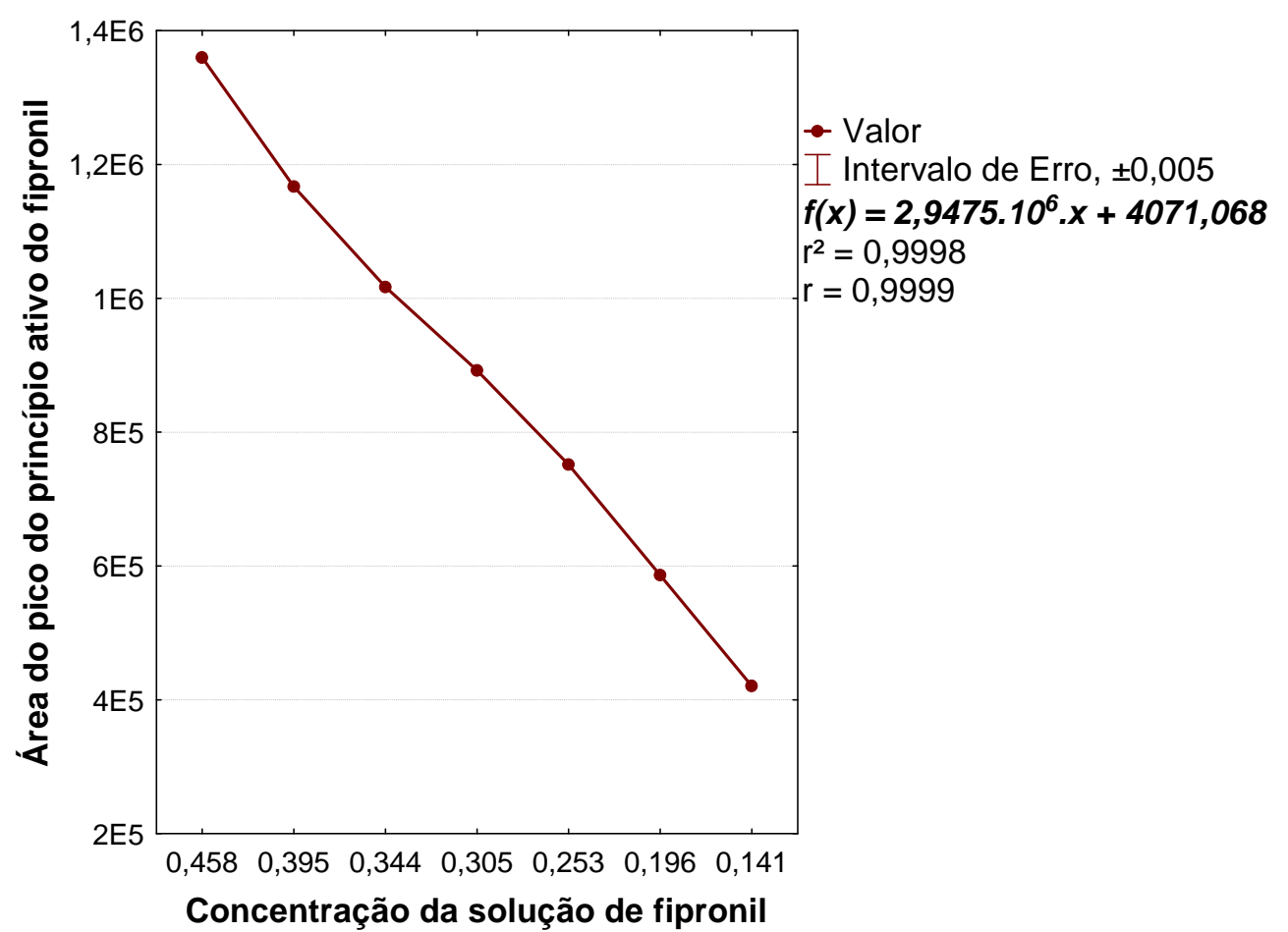

FIGURA 2 - Dispersão de pontos da área dos picos referentes ao princípio ativo do Fipronil em função da concentração da solução testada de Fipronil.

Fonte: Autores, (2019).

A determinação dos valores de LD e LQ para o princípio ativo de Fipronil originou-se da curva analítica obtida da regressão, apresentando ajuste de $99,98 \%$, e valor de desvio padrão residual, DPR, de 0,3705 . Relacionando os valores nas Equações 1 e 2 obteve-se, respectivamente, os valores de detecção de $0,00004 \%$ e quantificação de $0,00012 \%$ expressando os limites de detecção e quantificação em termos de concentração os valores de $0,128 \mathrm{mg} \cdot \mathrm{L}^{-1}$ e $0,426 \mathrm{mg} \cdot \mathrm{L}^{-1}$, respectivamente.

Resultados similares para os valores de LD e $L Q$ neste estudo foram encontrados por Goutal et al. (2016) em estudo de validação de método analítico para determinação de rifampicina por HPLC empregando detector UV.

A seletividade do método aplicada aos placebos não evidenciou picos na região de absorção do Fipronil pesquisado no tempo avaliado indicado a ausência de interferentes ou impurezas no material. Para os testes de recuperação soluções de diferentes concentrações do princípio ativo, p.a., de Fipronil foram preparadas e testadas em comparação com placebos obtendo as porcentagens de concentração determinada e a recuperação calculada. Os valores são apresentados na Tabela 2.

TABELA 2- Valores de concentração determinadas para o princípio ativo, massa de princípio ativo, p.a., e placebo em conjunto com a recuperação obtida.

\begin{tabular}{ccccc}
\hline $\begin{array}{c}\text { p.a. teórico } \\
(\%)\end{array}$ & $\begin{array}{c}\text { Massa de } \\
\text { p.a. }(\mathbf{m g})\end{array}$ & $\begin{array}{c}\text { Massa do } \\
\text { placebo } \mathbf{( m g})\end{array}$ & $\begin{array}{c}\text { Concentração } \\
\text { determinada (\%) }\end{array}$ & $\begin{array}{c}\text { Recuperação } \\
(\%)\end{array}$ \\
\hline 17,49 & 18,2 & 39,6 & 30,451 & 100,62 \\
16,05 & 16,7 & 45,2 & 25,860 & 99,73 \\
15,67 & 16,3 & 34,7 & 30,706 & 99,96 \\
14,99 & 15,6 & 33,1 & 30,522 & 99,14 \\
\hline
\end{tabular}




\begin{tabular}{lllll}
\hline 16,05 & 16,7 & 43,7 & 26,797 & 100,84 \\
16,24 & 16,9 & 34,6 & 31,613 & 100,23 \\
15,38 & 16,1 & 34,8 & 30,400 & 100,43 \\
30,56 & 31,8 & 19,7 & 59,528 & 100,31 \\
30,08 & 31,3 & 25,3 & 53,422 & 100,51 \\
30,85 & 32,1 & 22,0 & 57,255 & 100,40 \\
30,56 & 31,8 & 15,0 & 65,543 & 100,36 \\
32,68 & 34,0 & 22,3 & 57,890 & 99,74 \\
30,47 & 31,7 & 22,1 & 57,077 & 100,79 \\
32,68 & 34,1 & 22,1 & 58,348 & 100,17 \\
45,27 & 47,1 & 6,7 & 83,513 & 99,25 \\
47,19 & 49,1 & 6,8 & 84,620 & 100,24 \\
48,82 & 50,8 & 8,2 & 82,807 & 100,07 \\
43,44 & 45,2 & 7,3 & 83,169 & 100,51 \\
44,21 & 46,0 & 7,6 & 82,789 & 100,37 \\
44,40 & 46,2 & 7,1 & 83,004 & 99,64 \\
44,79 & 46,6 & 7,1 & 83,659 & 100,31 \\
\hline
\end{tabular}

A repetitividade que engloba a precisão inerente aos experimentos foi determinada para as soluções preparadas e medidas pelo mesmo analista. $\mathrm{O}$ valor do CV calculado foi de 0,237\%, estando abaixo do limite de $5 \%$ estipulado pela Resolução № 166 da ANVISA vigente.

Os valores do teste de Grubbs, $G_{1}$ e $G_{n}$, foram respectivamente 1,809 e 1,284 , indicando a concentração da distribuição estatística do teste e sua adequação aos dados bem como indicando a ausência de valores dispersos que fragilizam o modelo de regressão conforme apresentado no trabalho de Monteiro et al., 2018. Uma vez que se mostram inferiores aos valores tabelados no teste para 14 medições. A Tabela 3 apresenta os valores de concentração definidos na repetitividade, bem como os parâmetros estatísticos descritivos dos valores.

TABELA 3- Valores de massa, concentração de Fipronil determinada em duplicata e parâmetros estatísticos para a repetitividade e precisão inter-corridas.

\begin{tabular}{cccc}
\hline Amostra & Massa $(\mathbf{m g})$ & $\begin{array}{c}\text { Concentração } \\
\text { de Fipronil }(\%)\end{array}$ & $\begin{array}{c}\text { Parâmetros } \\
\text { estatísticos }\end{array}$ \\
\hline 01 & 58,9 & 44,897 & Média $=44,856 \%$ \\
02 & 60,1 & 44,852 & 44,670 \\
Desvio-padrão $=$ \\
03
\end{tabular}


A determinação da reprodutibilidade considera as medições realizadas com os erros sistemáticos, nesse caso verificados por medições conduzidas por analistas distintos e com metodologias análogas aquela descrita para a seletividade. Dessa forma cada uma das sete amostras preparadas foi medida em duplicata por dois analistas distintos, perfazendo 28 dados amostrados, 14 de cada analista.

Dos valores medidos no teste de precisão intermediária a média da concentração de Fipronil e desvio-padrão foram respectivamente $44,761 \%$ e $0,174 \%$ que conduziram para um Teste de Grubbs com $G_{1}$ de 1,861 e $G_{n}$ de 1,602, como os valores mostraram-se menores do que aquele tabelado para o teste de 28 medições, $G=2,876$, não se observou valores dispersos ou extremos, indicando a boa adequação do modelo aos dados não apresentando variabilidade aleatória e tendenciosa dos dados.

O valor de CV para os dados resultou em 0,388\%, evidenciando a homogeneidade das variâncias obtidas e a boa precisão intermediária conforme explicam Aguiar Júnior et al., (2018).

A estabilidade do método analítico testado, avaliado em termos do início e fim do estudo apresentou para as medições realizadas um desvio-padrão dos dados de 0,504\% e DPR de $0,509 \%$. No estudo de Bazilio et al., (2012) os valores mínimos de aceitabilidade do desvio-padrão e DPR em um modelo otimizado de validação se encontram acima daqueles obtidos no presente trabalho corroborando para a boa estabilidade verificada nesta metodologia.

Considerando a robustez do método avaliada para as variações de temperatura do forno em $\pm 5^{\circ} \mathrm{C}$ as medições conduzidas das sete soluções anteriormente preparadas para o teste de repetitividade são apresentadas na Tabela 4 juntamente com os parâmetros estatísticos.

TABELA 4- Valores de concentração do Fipronil em diferentes temperaturas e parâmetro estatísticos para verificação da robustez do método analítico.

\begin{tabular}{ccccc}
\hline $\begin{array}{c}\text { Amostr } \\
\text { a }\end{array}$ & $\begin{array}{c}\text { Concentração } \\
\text { em 20 } \mathbf{0}^{\circ} \mathbf{C} \text { (\%) }\end{array}$ & $\begin{array}{c}\text { Concentração } \\
\text { em 25 } \mathbf{5}^{\circ} \mathbf{C} \text { (\%) }\end{array}$ & $\begin{array}{c}\text { Concentração } \\
\text { em 30 } \mathbf{3 0}^{\circ} \mathbf{C} \text { (\%) }\end{array}$ & $\begin{array}{c}\text { Parâmetros } \\
\text { estatísticos }\end{array}$ \\
\hline 1 & 44,902 & 44,897 & 44,516 & Média $=44,805 \%$ \\
2 & 44,703 & 44,670 & 44,369 & \\
3 & 44,841 & 44,737 & 44,515 & Desvio-padrão $=$ \\
4 & 44,998 & 44,993 & 44,788 & $0,172 \%$ \\
5 & 44,978 & 44,957 & 44,731 & \\
6 & 44,966 & 44,903 & 44,715 & DPR $=0,110 \%$ \\
7 & 44,956 & 44,910 & 44,865 & \\
\hline
\end{tabular}

Os valores observados para a robustez do método apresentaram um valor médio de $44,805 \%$ com desvio-padrão de $0,172 \%$ e DPR de $0,110 \%$. Os parâmetros confirmam a boa estabilidade e previsibilidade dos valores que incrementam a robustez da metodologia avaliada apresentando os valores analisados para o desempenho da robustez do método correntes para aqueles limites apresentados pelo INMETRO conforme DOQ-CGCRE-008 revisada em 2016. 


\section{CONCLUSÃO}

O intervalo de 0,141 a $0,458 \mathrm{mg} \cdot \mathrm{mL}^{-1}$ de concentração do Fipronil avaliado mostrou-se linear e consonante com 0 indicado pelos órgãos reguladores de validação metodológica, INMETRO e ANVISA, para os coeficientes de determinação e correlação, respectivamente 0,9998 e 0,9999 , bem ajustados.

Os limites de detecção (LD) e quantificação (LQ) conduzidos em valores relativos e convertidos na concentração apresentaram os valores de $0,128 \mathrm{mg}^{-1} \mathrm{~L}^{-1} \mathrm{e}$ $0,426 \mathrm{mg} \cdot \mathrm{L}^{-1}$, respectivamente com uma imprecisão de $\pm 0,098 \%$. A recuperação do princípio ativo do Fipronil determinada no trabalho foi consonante aquela observada nos trabalhos com HPLC/UV.

A precisão e a repetitividade do método analítico em estudo apresentaram como desvio-padrão e DPR os valores de $0,106 \%$ e $0,237 \%$, respectivamente, ambos inseridos no limite de aceitabilidade determinado na RDC $n^{\circ} 166$ da ANVISA que regulamenta os testes de validação. A precisão intermediária com valores de 0,174\% para o desvio-padrão e $0,388 \%$ de DPR, também se mostrou dentro dos parâmetros fixados.

Os parâmetros de robustez e estabilidade apresentaram valores de média, desvio-padrão e DPR de 44,80\%, 0,172\% e 0,110\%, respectivamente. Esses valores também estão em consonância aos valores reportados na literatura para metodologias similares, comprovando assim a validação da metodologia analítica para quantificação do Fipronil por CLAE com detector UV.

\section{REFERÊNCIAS}

AGUIAR JÚNIOR, C.A.S.; SILVA, N.; SOARES, D.F.; FARIA, A.M. Solid-phase extraction of pesticide residues from surface water using a lab-made lower-retention sorbent. Química Nova, v.41, n.6, p.641-647, 2018. Disponível em: http://www.scielo.br/pdf/qn/v41n6/0100-4042-qn-41-06-0641.pdf DOI: $10.21577 / 0100-4042.20170223$

BARROS NETO, B.; PIMENTEL, M.F.; ARAÚJO, M.C.U. Recomendações para calibração em química analítica- Parte I. Fundamentos e calibração com um componente (calibração univariada). Química Nova, v.25, n.5, p.856- 865, 2002. Disponível em: http://www.scielo.br/pdf/qn/v25n5/11419.pdf DOI: 10.1590/S010040422002000500024

BAZILIO, S.F.; BOMFIM, J.V.M.; ALMEIDA, J.R.; ABRANTES, P.M.S. Uso de planilha eletrônica na verificação da adequação de curva analítica ao modelo linear. Analytica, São Paulo, v.10, n.59, p.60-67, jun./jul. 2012. Disponível em: https://www.arca.fiocruz.br/bitstream/icict/9141/2/analytica_59_60-67.pdf Acesso em 01/04/2019.

BENEVIDES, C.M.J.; BEZERRA, M.A.; PEREIRA, P.A.P.; ANDRADE, J. B. HSSPME/ GC-MS analysis of VOC and multivariate techniques applied to the discrimination of Brazilian varieties of mango. American Journal of Analytical Chemistry, v.5, n.3, p.157-164, 2014. Disponível em: https://file.scirp.org/Html/22200795_42927.htm DOI: 10.4236/aiac.2014.53019 
BRASIL. Agência Nacional de Vigilância Sanitária - ANVISA. Resolução da Diretoria Colegiada $n^{\circ} 166$, de 24 de julho de 2017. Estabelece critérios para a validação de métodos analíticos. Diário Oficial da República Federativa do Brasil, Brasília, DF, 2017.

BRASIL, Instituto Nacional de Metrologia, Normalização e Qualidade Industrial INMETRO. DOQ-CGCRE-008, de 05 de agosto de 2016. Orientação Sobre Validação de Métodos Analíticos. Disponível em: http://www.inmetro.gov.br/Sidoq/Arquivos/CGCRE/DOQ/DOQ-CGCRE-8_05.pdf. Acesso em 01/04/2019.

CASSINI, S. T. A; ANTUNES; P. W. P.; KELLER, R. Validação de método analítico livre de acetonitrila para análise de microcistinas por cromatografia líquida de alta eficiência. Química Nova, v.36 n.8, p.1208-1213, 2013. Disponível em: http://www.scielo.br/pdf/qn/v36n8/v36n8a19.pdf DOI: 10.1590/S010040422013000800019

CANDIOTI, L.V.; ZAN, M.M.; CAMARA, M.S.; GOICOECHEA, H.C. Experimental design and multiple response optimization. Using the desirability function in analytical methods development. Talanta, v.124, p.123-138, 2014. Disponível em: https://www.sciencedirect.com/science/article/pii/S0039914014000459 DOI: 10.1016/j.talanta.2014.01.034

CHAWLA, S.; PATEL, H.K.; VAGHELA, K.M.; PATHAN, F.K.; GOR, H.N.; PATEL, A.R.; SHAH, P.G. Development and validation of multiresidue analytical method in cotton and groundnutoil for 87 pesticides using low temperature and dispersive cleanup on gas chromatography and liquid chromatography-tandem mass spectrometry. Bioanal Chem, n. 408, p.983-997, 2016. Disponível em: https://www.ncbi.nlm.nih.gov/pubmed/26660874 DOI: 10.1007/s00216-015-9192-2

CHELLINI, P.R.; LAGES, E.B.; FRANCO, P.H.C.; NOGUEIRA, F.H.A.; CÉSAR, I.C.; PIANETTI, G.A. Development and validation of an HPLC method for simultaneous determination of rifampicin, isoniazid, pyrazinamide, and ethambutol hydrochloride in pharmaceutical formulations. Journal of AOAC International, v.98, p.1234-1239, 2015. Disponível em: https://www.ncbi.nlm.nih.gov/pubmed/26525241 DOI: 10.5740/jaoacint.14-237.

COELHO, E.R.C.; LEAL, W.P.; SOUZA, K.B.D.; ROZÁRIO, A.D.; ANTUNES, P.W.P. Development and validation of analytical method for analysis of 2, 4-D, 2, 4-DCP and 2, 4, 5-T for monitoring of public water supply. Engenharia Sanitária e Ambiental, v.23, p.1043-1051, $2018 . \quad$ Disponível em: http://www.scielo.br/pdf/esa/v23n6/1809-4457-esa-23-06-1043.pdf DOI: $10.1590 / \mathrm{s} 1413-41522018161536$

DURAND, E.; LECOMTE, J.; VILLENEUVE, P. From green chemistry tonature: the versatile role of low transition temperature mixtures. Biochimie, v.120, p.119-123, 2016 . 
https://www.sciencedirect.com/science/article/pii/S0300908415002941

DOI:

10.1016/j.biochi.2015.09.019

GOUTAL, S.; AUVITY, S.; LEGRAND, T.; HAUQUIER, F.; CISTERNINO, S.; CHAPY, H.; SABA, W.; TOURNIER, N. Validation of a simple HPLC-UV method for rifampicin determination in plasma: Application to the study of rifampicin arterio venous concentration gradient. $\mathbf{J}$ Pharm Biomed, n. 123, p.173-178, 2016. Disponível em: https://www.ncbi.nlm.nih.gov/pubmed/26907700 DOI: 10.1016/j.jpba.2016.02.013.

GUPTA, R.C. \& ANADÓN, A. Chapter 42 - Fipronil. Veterinary Toxicology (Third Edition), p.533-538, 2018. Disponível em: https://www.sciencedirect.com/science/article/pii/B9780128114100000428 DOI: 10.1016/B978-0-12-811410-0.00042-8

GOES JUNIOR, E.J.A.; ROEDER, J. S.; OLIVEIRA, K. B.; FERREIRA, M. P.; SILVA, J. G. D. Validation of a spectro photometric method for quantification of acetyl salicylic acid in pharmaceutical formulations: a proposal of experimental activity for instrumental analysis. Química Nova, v.42, p.99-104, 2019. Disponível em: http://www.scielo.br/pdf/qn/v42n1/0100-4042-qn-42-01-0099.pdf DOI: 10.21577/0100-4042.20170300

HOLDER, P.J.; JONES, A.; TYLER, C.R.; CRESSWELL, J.E. Fipronil pesticide as a suspect in historical mass mortalities of honeybees. Proceedings of the National Academy of Sciences, v.115, p.13033-13038, 2018. Disponível em: https://www.pnas.org/content/115/51/13033 DOI: 10.1073/pnas.1804934115

IAMMARINO, M.; MARINO, R.; ALBENZIO, M. Howmeaty? Detection and quantification of adulterants, foreign proteins and food additives in meat products. International Journal of Food Science \& Technology, v. 52, p.851-863, 2017. Disponível em: https://onlinelibrary.wiley.com/doi/epdf/10.1111/ijfs.13350 DOI: 10.1111/ijfs. 13350

INMETRO - Instituto Nacional de Metrologia, Normalização e Qualidade Industrial (INMETRO); Orientações sobre Validação de Métodos de Ensaios Químicos, DOQCGCRE-008, 2018.

LEGRAND, P.; DESDION, A.; BOCCADIFUOCO, G.; WOJCICLI, D.A. et al. Development of an HPLC/UV method for the evaluation of extractables and leachables in plastic: Application to a plastic-packaged calcium gluconate glucoheptonate solution. Journal of Pharmaceutical and Biomedical Analysis, v.155, p.298-305, 2018. Disponível em: https://www.science direct.com/science/article/abs/pii/S0731708517331448 10.1016/j.jpba.2018.03.044

LUCIANI-GIACOBBE, L.C.; GUZMAN, M.L.; MANZO, R.H.; OLIVERA, M.E. Validation of a simple isocratic HPLC-UV method for rifampicin and isoniazid quantification in human plasma. Journal of Applied Pharmaceutical Science, v.8, p.093-099, 2018. Disponível em: 
http://www.japsonline.com/admin/php/uploads/2681_pdf.pdf

DOI: 10.7324/JAPS.2018.8715

MONTEIRO, R.T.; SANTAN, R.R.M.; SILVA, A.M.R.B.; LUCENA, A.L.A.; ZAIDAN, L. E.M.C.; SILVA, V.L.; NAPOLEÃO, D.C. Degradation of the pharmaceuticals nimesulide and ibuprofen using photo-Fentonprocess: toxicity studies, kinetic modeling and use of artificial neural networks. Electronic Journal of Management, Education and Environmental Technology (REGET), v.22, p.1-21, 2018. Disponível em: https://periodicos.ufsm.br/reget/article/view/31563DOI: $10.5902 / 2236117031563$

NOVAES, C.G.; YAMAKI, R.T.; DE PAULA, V.F.; NASCIMENTO J.B.B.; BARRETO, J.A.; VALASQUES, G.S.; BEZERRA, M.A. Otimização de Métodos Analíticos Usando Metodologia de Superfícies De Resposta - Parte I: Variáveis de Processo. Revista Virtual de Química, v.9, p.1184-1215, 2017. Disponível em: http://rvq.sbq.org.br/imagebank/pdf/NovaesNoPrelo.pdf $\quad$ DOI: $\quad 10.21577 / 1984-$ 6835.20170070

OHIRA, S.; KANEDA, K.; MATSUZAKI, T.; MORI, S.; MORI, M. et al. Universal HPLC Detector for Hydrophilic Organic Compounds by Means of Total Organic Carbon Detection. Analytical chemistry, v.90, n.11, p.6461-6467, 2018. Disponível em: $\quad$ https://pubs.acs.org/doi/ipdf/10.1021/acs.analchem.7b04849 DOI: 10.1021/acs.analchem.7b04849

QI, P.; LIN, Z.; CHEN, G.; XIAO, J.; LIANG, Z. et al. Fast and simultaneous determination of eleven synthetic color additives in flour and meat products by liquid chromatography coupled with diode-array detector and tandem mass spectrometry.

Food Chemistry, v.181, p.101-110, 2015. Disponível em: https://www.sciencedirect.com/science/article/pii/S0308814615002654 DOI: 10.1016/j.foodchem.2015.02.075

RIBANI, M.; BOTTOLI, C.B.G.; COLLINS, C.H.; JARDIM, I.C.S.F.; MELO, L.F.C. Validação em métodos cromatográficos e eletroforéticos. Química Nova, v.27, p.771780, 2004. Disponível em: http://www.scielo.br/pdf/\%0D/qn/v27n5/a17v27n5.pdf DOI:10.1590/S0100-40422004000500017

SANTOS, L.G.; PEIXOTO SOBRINHO, T.J.S.; CABRAL, D.L.V.; AMORIM, E.L.C. Validação de metodologia analítica para determinação de taninos pelo método de difusão radial. Revista Brasileira de Plantas Medicinais, v.16, n.4, p.881-885, 2014. Disponível em: http://www.scielo.br/pdf/rbpm/v16n4/a14v16n4.pdf DOI: 10.1590/1983-084X/12_023.

SANTOS, L.M.G. Avaliação das impurezas elementares do medicamento captopril comercializadas na cidade do Rio de Janeiro, Brasil. Revista Virtual de Química, v. 10, n. 3, 2018. Disponível em: http://rvq.sbq.org.br/imagebank/pdf/v10n3a19.pdf DOI: 10.21577/1984-6835.20180051 
SCHWEIKART, F.; HULTHE, G. HPLC-UV-MS analysis-asource for severe oxidation artifacts. Analytical chemistry, v.91, p.1748-1751, 2019. Disponível em: https://pubs.acs.org/doi/ipdf/10.1021/acs.analchem.8b05845 DOI: 10.1021/acs.analchem.8b05845

SWARTZ, M.E. \& IRA S.K. Analytical method development and validation. CRC Press, 2018. 